\title{
Hybrid Simulation Experience-Hybrid Simulator Model vs. Manikin in Bladder Catheterization Procedure: A Pilot Study
}

Sara Nikolic ${ }^{1}$, Mirjam Mocnik ${ }^{2}$ and Sebastjan Bevc ${ }^{3,4^{*}}$

${ }^{1}$ Clinic for Internal Medicine, Department of Gastroenterology, University Clinical Center Maribor, Slovenia

${ }^{2}$ Clinics for Pediatrics, University Clinical Center Maribor, Slovenia

${ }^{3}$ Clinics for Internal Medicine, Department of Nephrology, University Clinical Center Maribor, Slovenia

${ }^{4}$ Faculty of Medicine, University of Maribor, Slovenia

*Corresponding author: Sebastjan Bevc, Associate Professor, Department of Nephrology, Clinic for Internal Medicine, University Clinical Center Maribor and Faculty of Medicine, University of Maribor, Taborska 8, 2000 Maribor, Slovenia, Tel: + 0038623212485; E-mail: sebastjan.bevc@ukc-mb.si

Received date: August 14, 2017; Accepted date: September 26, 2017; Published date: September 29, 2017

Copyright: (C) 2017 Nikolic S, et al. This is an open-access article distributed under the terms of the Creative Commons Attribution License, which permits unrestricted use, distribution, and reproduction in any medium, provided the original author and source are credited.

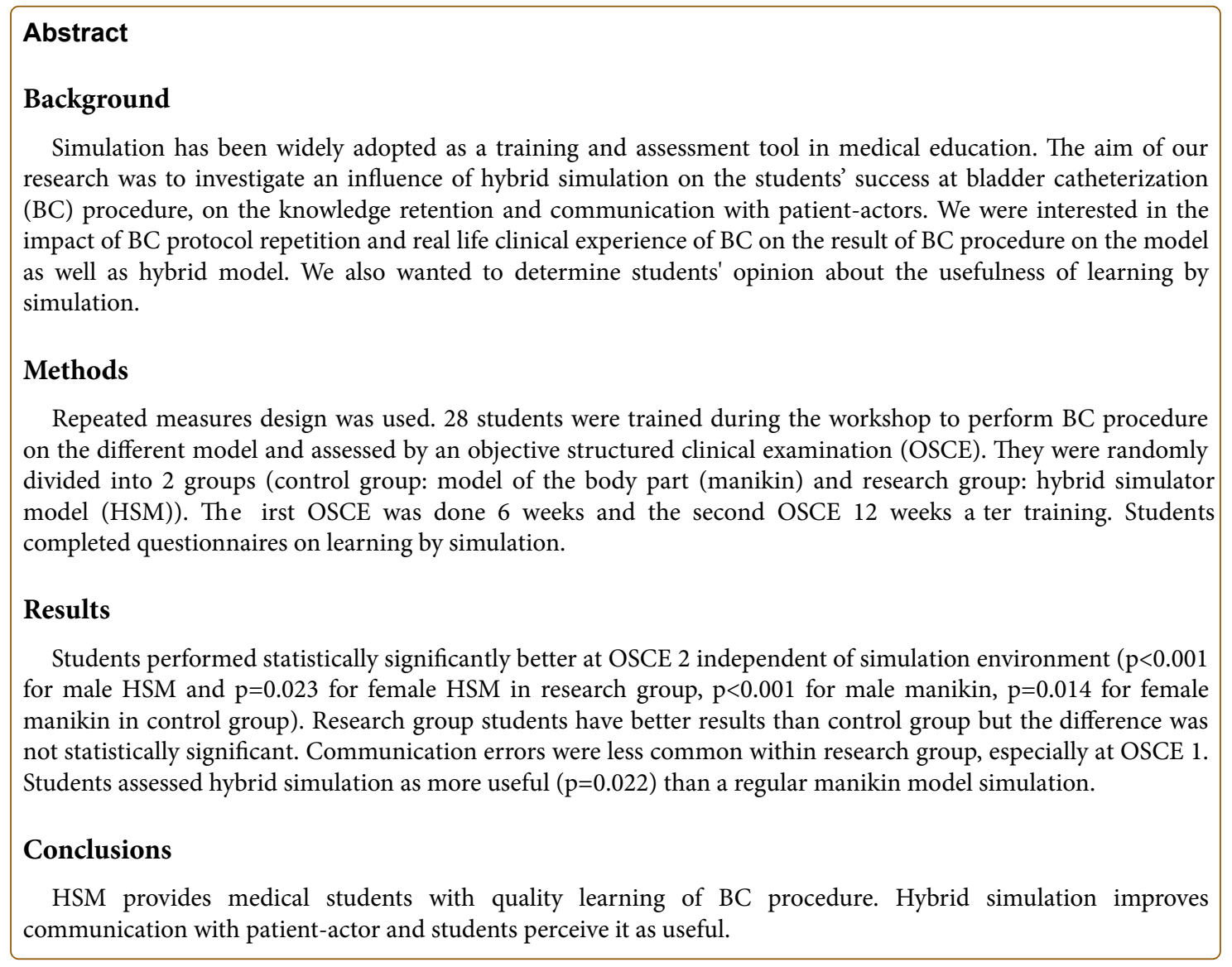

Keywords: Simulation; Learning; Bladder catheterization procedure; Manikin; Hybrid model

\section{Abbreviations: \\ BC: Bladder Catheterization; HSM: Hybrid Simulator Model; OSCE: Objective Structured Clinical Examination; C: Communication; m: Male; f: Female.}

\section{Introduction}

Simulation based education is a branch of simulation technology which uses different approaches to simulate clinical situations with the purpose of active clinical skills learning. The theoretical basis for learning by simulation is the constructivist model of learning which states that learning is the result of active participation in the process of acquiring knowledge and not just passive reception of information. The constructivist learning model is characterized by better knowledge retention, faster transfer in the learning process and the flattening of the curve of forgetting [1]. Constructivist model compared to the traditional model is more effective in the field of learning with 
simulation. Also, there is evidence of increased complexity of thinking [2].

Simulation manikins and sophisticated human patient simulators (HPS) are recognized worldwide as an effective way of learning clinical skills and are an obligatory part of modern medical curriculum [3]. In contrast to clinical experience, error in a simulated environment is allowed and with practice eliminated. Consequently, when encountered with real life patients, students are equipped with skills and self-confidence and their transition to clinics is mellower and more successful. Also, when having proper equipment and imagination, medical simulation enables precise simulation of conditions that can be endlessly repeated anytime and anywhere [4]. A very significant level of skills transfer can be achieved with training through a structured supervised simulation syllabus. Simulation technology can improve patient safety [5].

Clinical simulation environment can be achieved in several ways. There are models of the human body, models of human body parts, simulated patients, computer-generated simulators and hybrid models - a combination of simulated patient and model of the human body [4]. Hybrid simulations expand the learning context and manage to achieve a holistic simulation by reinforcing the doctor patient communication. Skillful communication is one of the most essential tools for establishing a positive relationship between doctor and patient, allowing greater efficiency in diagnosis and treatment. During hybrid simulation students learn clinical skills along with communication with patients and not as two separate elements when working with patients [6,7]. Studies showed that with hybrid simulation students/doctors simultaneously acquire technical and communication skills [8]. Hybrid simulation improves both the communication skills of the student/doctor [9], and their learning experience (10). By setting up a holistic objective, hybrid simulation does not inhibit the acquisition of desired clinical skills [6]. In addition, hybrid simulator models allow different levels of communication requirements and enable individualized learning mode by adjusting the scenarios [11]. These results do not apply to all research. Posner and Hamstra showed that the simulation models and hybrid simulations are equivalent in acquisition of communication skills. On the other hand, communication with the simulated patient can interfere with obtaining technical skills [12].

We used two types of simulation in our research - simulation of the human body part (manikins) and hybrid simulation model for the bladder catheterization procedure. The primary aim of our research was to investigate an influence of hybrid simulation on the students' success at bladder catheterization procedure, on the knowledge retention and communication with patient-actors. Additionally, we were interested in students' perception of usefulness of hybrid simulation and their general satisfaction with it.

\section{Methods}

\section{Subjects}

The research project involved students attending 6th year of the Faculty of Medicine, University of Maribor, a total of 28 students sampled by opportunity sampling in academic year 2013/2014. They were randomly divided into two groups: hybrid simulator model (HSM) group $(\mathrm{N}=14)$ and control group $(\mathrm{N}=14)$. HSM consisted of human body part simulation model being between the legs of simulated patient (Figure 1). Control group performed on body part model (manikins) (Figure 2). After oral agreement, the informed consent form was signed by the participants. Ethical committee permission was not obligatory.

\section{Data collecting}

Students attended a clinical skills workshop as part of the Internal Medicine practicum, where they were taught the bladder catheterization (BC) procedure. Workshop was held once in the winter and once in the summer semester. An objective structured clinical examination (OSCE) 1 followed six weeks after the workshop. At OSCE 1 students performed the $\mathrm{BC}$ procedure twice, on a male and female manikins (Figure 1; Table 1) or HSM depending on whether they we part of a control or HSM group. Six weeks after, the OSCE 1 was followed by OSCE 2, in which students were performing BC procedure, on the same simulation modality as in OSCE 1 . BC procedure checklist for male model had the maximum of 32 points, and the female 31 . In both cases there was a time limit of 10 minutes to perform the catheterization. No points were assigned after the time limit.

\section{Data processing}

Statistical analysis was performed with SPSS Statistics 19. As part of the descriptive statistical analysis we determined following parameters: minimum and maximum result, the average and standard deviation of points scored and the time needed for the completion of the procedure. All these values were calculated for all students together and for each group separately. Also, we performed a comparison of averages between the two groups with respect to the time required for $\mathrm{BC}$ procedure and the result achieved in the OSCE with the test of independent samples. Statistical significance was set at $\mathrm{p}$-value $<0.05$. We also analyzed the responses of the questionnaire. Finally, we counted the most common mistakes for OSCE 1 and 2 on male and female model and compare the dynamics of the frequency of errors in the manikin model and HSM.

\section{Results}

Table 1 presents descriptive statistics (minimum, maximum, mean and standard deviation) for the control and research group as well as the comparison of OSCE 1 and 2 outcomes (score and time). There was a statistically significant improvement in OSCE score in male and female model (manikin and HSM) in the OSCE 2, while the difference in the time required for the $\mathrm{BC}$ procedure was not statistically significant.

Research group scored higher mean scores in both OSCE 1 and OSCE 2 (Figure 3). However, despite higher mean score, no statistically significant difference between control and research group neither in OSCE 1 nor in OSCE 2 was found (Table 2).

We have counted errors occurred during the OSCE 1 and OSCE 2 (Tables 3 and 4). There were two types of errors we have put special emphasis on: technical and communication errors. Most common communication errors were putting the patient-actor into comfortable position before and after the BCP as well as inquiring about the pain level during the catheterization and inflation of the catheters balloon. When comparing HSM and manikin, we found that students made less communication errors at HSM during OSCE 1. On the other hand at OSCE 2, these differences were not detected. 
Finally, perception of usefulness in different types of simulation on a Linkert scale from 1-5 (1 - not useful at all, 5 - very useful) is showed in Figure 4. We found a statistically significant difference between control and research group $(\mathrm{p}=0.022)$.

\section{Discussion}

The primary goal of medical school is to provide the future doctors with competencies, which will suffice for high-quality patient treatment. The general purpose of research in the field of medical education is to search for most efficient and economic ways of education. Improved outcomes in treatment of patients directly rely on the mode of education [12]. Our study sought to identify the effectiveness of teaching $\mathrm{BC}$ procedure by hybrid simulation. The effectiveness was measured by success at OSCE, retention of knowledge, number of communication errors with patient-actors during OSCE and the self-evaluation of the usefulness of hybrid simulation.

In the control and the research group there was a statistically significant improvement in OSCE score, while significant differences in the time required for the completion of $\mathrm{BC}$ procedure were not found. Students of both study groups 6 weeks after OSCE 1, equipped with the feedback and their assessors, inserted bladder catheter better at OSCE 2 and took enough time to do so. Performance results OSCE 2 coincided with the results of the Dutch study in 2011, which showed that deliberate practice on simulators has positive effects on learning clinical skills [13].

Despite the fact that research group was more successful at both OSCEs, the difference in the mean OSCE score was not statistically significant. Sample size of our study was small and probably more participants would generate statistical significance. It was suggested that the presence of the patient-actor improves communication steps in the protocol, however, it can also reduce the technical skills and concentration of the student, thus research group students would not score significantly better. In fact, that explanation is supported by study of Posner et al., who simulated gynecological examination in hybrid simulation using different friendly and talkative patients-actors. They found that friendly and very talkative patients-actors reduced the technical skill and concentration of the students [12].

Students made most errors at the level of communication. Communication points represent about a quarter of our protocol. Improving outcomes of OSCEs were associated with improvement in communication with the patient-actor. Our analysis showed that students put more emphasis on sterility areas when they see a patientactor in front of them. Error in the handling of the foreskin was the only one who appeared frequently in both OSCEs and we associated it with the feature of our model (penis without retractable foreskin).

The purpose of the advanced simulations is to raise the efficiency in acquiring and retention of knowledge for the sake of future clinical practice. Study by Lo et al. from 2011 found that high-fidelity simulation in learning advanced cardiac life support is more effective, but the retention of knowledge after one year was the same as with traditional learning [14]. We did not test knowledge retention after one year, because this was not possible due to the study schedule of 6 th year medical school students. However, examinations were conducted after 6 and 12 weeks (OSCE 1 and 2) and we found that the students performed better after 12 than 6 weeks. This could be interpreted by the fact that short time had passed between the examinations and the $\mathrm{BC}$ procedure was not forgotten. For the future investigations, it would be useful to determine if the hybrid simulation allows better retention of knowledge even after one year.

A very important factor in learning is general satisfaction with the way students learn. Research group students have evaluated learning with simulation as more useful compared to students in the control group. The difference was statistically significant. Our findings are supported by the conclusions of the study by Raymond et al. from 2009 focused on comparing the simulation to the group discussion when teaching emergency medicine. They associated better learning outcomes with the popularity of simulation among students [15].

The small sample of volunteers participating is a main weakness of our study. Between the two OSCEs some students dropped out. Another disadvantage of our study is predominant quantitative assessment of both technical and communication skills. On the other hand patients - actors could have assessed the students by patient perception score (PPS). Nowadays, cutting edge technology enables puppets to "speak", but a large survey of physicians and midwives (Fire drill Simulation and Evaluation (Safe)) did not confirm the advantages of learning with advanced technology and high-fidelity simulation over the hybrid simulation. Therefore, more accurate assessment could replace learning with complex simulators [16].

The advantage of our research is a good bias, because the students were assessed by OSCE, the assessment was carried out by experienced peer tutors and patients-actors were precisely instructed how to behave. Via a questionnaire, we summarized the students' opinion about the $\mathrm{BC}$ procedure simulation. If peer tutors assess using a checklist, they are equivalent to experienced clinicians; therefore peertutor assessment was not questionable [17]. However, we would tend to educate the patient-actors in future studies, because research has shown a significant influence of the patient-actors training on the performance of a clinical skill [18].

The goal of our research project was to determine how hybrid simulation influences the learning process of $\mathrm{BC}$ procedure.

The results of our study has shown that students preformed BC procedure statistically better the second time, whatever the simulation surroundings. Research group had slightly better results, but no statistically significant differences were found. Fewer communication errors were found in the research group only during OSCE 1 . Students felt that learning by simulation is very useful, especially the hybrid simulation.

The hybrid simulation allows a better simulation scenario for medical students during $\mathrm{BC}$ procedure learning, which is a demanding clinical skill where communication with patients is often neglected. Our results encourage further research of hybrid simulation and its impact on the technical and communication skills as well its consequences on long-term retention of knowledge.

\section{Conflicts of Interest}

No conflict of interest, no specific funding. 
Citation: Nikolic S, Mocnik M, Bevc S (2017) Hybrid Simulation Experience-Hybrid Simulator Model vs. Manikin in Bladder Catheterization Procedure: A Pilot Study. J Health Educ Res Dev 5: 235. doi:10.4172/2380-5439.1000235

Page 4 of 6

\begin{tabular}{|c|c|c|c|c|c|c|c|}
\hline & & & \multicolumn{4}{|l|}{ Score (points) } & \\
\hline \multirow{12}{*}{$\begin{array}{l}\text { Control group } \\
(\mathrm{N}=14)\end{array}$} & OSCE 1 & \multirow{5}{*}{$o^{\lambda}$ model } & 15 & 28 & 22.4 & \pm 3.5 & \multirow{2}{*}{0.001} \\
\hline & OSCE 2 & & 21 & 31 & 26.8 & \pm 2.6 & \\
\hline & & & Time (s) & & & & \\
\hline & OSCE 1 & & 241 & 564 & 349.9 & \pm 88.0 & \\
\hline & OSCE 2 & & 180 & 598 & 298.4 & \pm 103.9 & \\
\hline & & & Score (points) & & & & \\
\hline & OSCE 1 & & 14 & 27 & 22.2 & \pm 3.8 & \\
\hline & OSCE 2 & & 20 & 31 & 26.1 & \pm 3.3 & \\
\hline & & i model & Time (s) & & & & \\
\hline & OSCE 1 & & 257 & 457 & 334.1 & \pm 63.6 & \\
\hline & OSCE 2 & & 221 & 437 & 315.1 & \pm 63.4 & \\
\hline & & & Score (points) & & & & \\
\hline & OSCE 1 & & 15 & 32 & 24.4 & \pm 4.2 & \\
\hline & OSCE 2 & & 22 & 32 & 28.1 & \pm 3.0 & 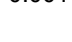 \\
\hline & & medel & Time (s) & & & & \\
\hline & OSCE 1 & & 203 & 522 & 391.6 & \pm 96.7 & \\
\hline & OSCE 2 & & 230 & 478 & 306.9 & \pm 64.7 & \\
\hline $\begin{array}{l}\text { Research } \\
\text { group }(N=14)\end{array}$ & & & Score (points) & & & & \\
\hline & OSCE 1 & & 16 & 31 & 24.3 & \pm 3.4 & \\
\hline & OSCE 2 & & 19 & 31 & 27.4 & \pm 3.4 & \\
\hline & & q model & Time (s) & & & & \\
\hline & OSCE 1 & & 272 & 570 & 385.9 & \pm 79.7 & \\
\hline & OSCE 2 & & 219 & 530 & 350.5 & \pm 78.0 & \\
\hline
\end{tabular}

Table 1: Descriptive statistics and comparison of OSCE 1 and OSCE 2 outcomes (score and time) in control and research group. Statistical significance is acquired when $\mathrm{p}<0.05$.

\begin{tabular}{|c|c|c|}
\hline & $p$-value & \\
\hline Control group vs Research group & OSCE 1 & OSCE 2 \\
\hline score: $\hat{\partial}$ model & 0.17 & 0.2 \\
\hline time: $\widehat{\jmath}$ model & 0.435 & 0.238 \\
\hline score: $\$$ model & 0.128 & 0.279 \\
\hline
\end{tabular}

\begin{tabular}{|l|l|l|}
\hline time: $q$ model & 0.071 & 0.239 \\
\hline
\end{tabular}

Table 2: Comparison of control and research group outcomes (score and time) in OSCE 1 and OSCE 2. Statistical significance is acquired when $\mathrm{p}<0.05$.

\begin{tabular}{|c|c|c|c|c|c|c|c|}
\hline Number of errors & & Position 1 (C) & Sterility & Foreskin 1 & Pain 1+2 (C) & Foreskin 2 & Position 2 (C) \\
\hline \multirow{2}{*}{ manikin } & OSCE 1 & 9 & 11 & 2 & 15 & 12 & 12 \\
\hline & OSCE 2 & 6 & 6 & 7 & 6 & 7 & 6 \\
\hline $\mathrm{HSM}$ & OSCE 1 & 8 & 6 & 7 & 10 & 12 & 10 \\
\hline
\end{tabular}


Citation: Nikolic S, Mocnik M, Bevc S (2017) Hybrid Simulation Experience-Hybrid Simulator Model vs. Manikin in Bladder Catheterization Procedure: A Pilot Study. J Health Educ Res Dev 5: 235. doi:10.4172/2380-5439.1000235

Page 5 of 6

\begin{tabular}{|l|l|l|l|l|l|l|l|}
\hline & OSCE 2 & 3 & 3 & 7 & 5 & 10 & 6 \\
\hline
\end{tabular}

Table 3: Most common mistakes made at male manikin and hybrid simulator model (HSM) during OSCE 1 and OSCE 2. Position 1: comfortable patient-actor`s position before BC procedure; Position 2: comfortable patient-actor`s position after BC procedure; Sterility: sterile gloving technique, conserving right hand sterile during the $\mathrm{BC}$ procedure; Foreskin 1: pulling back of the foreskin; Foreskin 2: pulling up of the foreskin; Pain 1: enquiring about pain during catheter insertion; Pain 2: enquiring about pain during balloon inflation; C: abbreviation for errors in communication steps of the protocol.

\begin{tabular}{|l|l|l|l|l|l|l|}
\hline $\begin{array}{l}\text { Number } \\
\text { of errors }\end{array}$ & & $\begin{array}{l}\text { Position 1 } \\
\text { (C) }\end{array}$ & Sterility & Pain 1 & $\begin{array}{l}\text { Pain 2 2 } \\
\text { (C) }\end{array}$ & $\begin{array}{l}\text { Position 2 } \\
\text { (C) }\end{array}$ \\
\hline \multirow{2}{*}{ o manikin } & OSCE 1 & 11 & 8 & 7 & 7 & 12 \\
\cline { 2 - 7 } & OSCE 2 & 3 & 8 & 2 & 5 & 5 \\
\hline \multirow{2}{*}{ \% HSM } & OSCE 1 & 6 & 8 & 6 & 5 & 11 \\
\cline { 2 - 7 } & OSCE 2 & 3 & 3 & 3 & 4 & 8 \\
\hline
\end{tabular}

Table 4: Most common mistakes made at female manikin and hybrid simulator model (HSM) during OSCE 1 and OSCE 2. Position 1: comfortable patient-actor's position before BC procedure; Position 2: comfortable patient-actor's position after BC procedure; Sterility: sterile gloving technique, conserving right hand sterile during the $\mathrm{BC}$ procedure; Pain 1: enquiring about pain during catheter insertion; Pain 2: enquiring about pain during balloon inflation; C: abbreviation for errors in communication steps of the protocol.

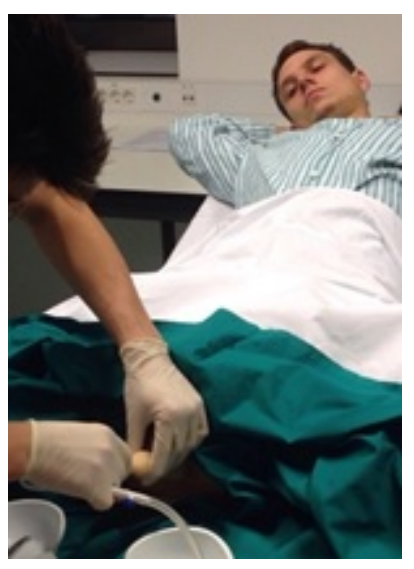

Figure 1: Hybrid simulator model. 
Citation: Nikolic S, Mocnik M, Bevc S (2017) Hybrid Simulation Experience-Hybrid Simulator Model vs. Manikin in Bladder Catheterization Procedure: A Pilot Study. J Health Educ Res Dev 5: 235. doi:10.4172/2380-5439.1000235

Page 6 of 6

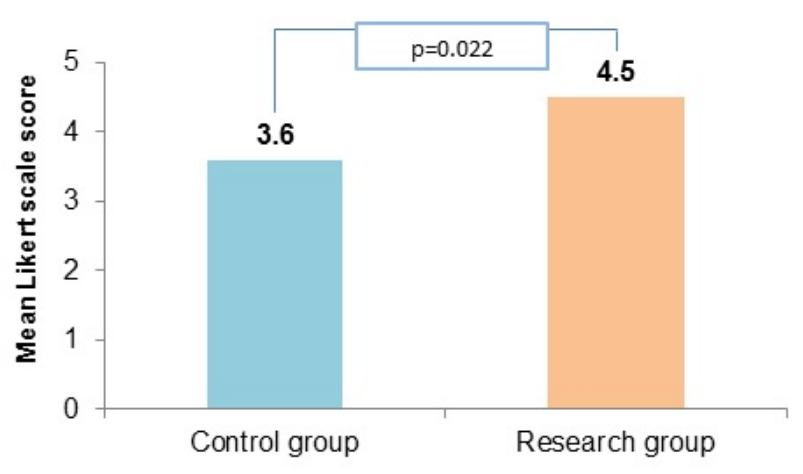

Figure 4: Self-evaluation of usefulness of simulation in control group (manikin simulation) and research group (hybrid simulation).

\section{References}

1. Bodemer D (2005) Enhancing Simulation-Based Learning through Active External Integration of Representations. Proceedings of the Twenty-Sixth Annual Conference of the Cognitive Science Society pp: 138-143.

2. Narli S (2011) Is constructivist learning environment really effective on learning and long-term knowledge retention in mathematics? Example of the infinity concept. Educational Research and Reviews 6: 36-49.

3. Bradley P (2006) The history of simulation in medical education and possible future directions. Medical Education 40: 254-262.

4. Weller JM, Nestel D, Marshall SD, Brooks PM, Conn JJ (2012) Simulation in clinical teaching and learning. The Medical Journal of Australia 196: $1-5$.

5. Pahor D (2014) Virtual reality simulator training - a new era in surgical education program. Acta Medico-Biotechnica 7: 7-8.

6. Brydges R, Mallette C, Pollex H, Carnahan H, Dubrowski A (2012) Evaluating the influence of goal setting on intravenous catheterization skill acquisition and transfer in a hybrid simulation training context.
Simulation in Healthcare: Journal of the Society for Simulation in Healthcare 7: 236-242.

7. Dyche L (2007) Interpersonal Skill in Medicine: The Essential Partner of Verbal Communication. Journal of General Internal Medicine 22: 1035-1039.

8. Stroud L, Cavalcanti RB (2013) Hybrid simulation for knee arthrocentesis: improving fidelity in procedures training. J Gen Intern Med 28: 723-727.

9. Siassakos D, Draycott T, O'Brien K, Kenyon C, Bartlett C, et al. (2010) Exploratory randomized controlled trial of hybrid obstetric simulation training for undergraduate students. Simulation in Healthcare: Journal of the Society for Simulation in Healthcare 5: 193-198.

10. Girzadas DV, Antonis MS, Zerth H, Lambert M, Clay L, et al. (2009) Hybrid simulation combining a high fidelity scenario with a pelvic ultrasound task trainer enhances the training and evaluation of endovaginal ultrasound skills. Academic Emergency Medicine 16: 429-435.

11. Kyaw Tun J, Granados A, Mavroveli S, Nuttall S, Kadiyala AN, et al. (2012) Simulating various levels of clinical challenge in the assessment of clinical procedure competence. Ann Emerg Med 60: 112-120.

12. Posner GD, Hamstra SJ (2013) Too much small talk? Medical students' pelvic examination skills falter with pleasant patients. Medical Education 47: 1209-1214

13. Duvivier RJ, van Dalen J, Muijtjens AM, Moulaert VR, van der Vleuten CP, et al. (2011) The role of deliberate practice in the acquisition of clinical skills. BMC Med Educ 11: 101.

14. Lo BM, Devine AS, Evans DP, Byars DV, Lamm OY, et al. (2011) Comparison of traditional versus high-fidelity simulation in the retention of ACLS knowledge. Resuscitation 82: 1440-1443.

15. Harrison's Principles of Internal Medicine (2008) New York, McGrawHill. p: 17.

16. Crofts JF, Bartlett C, Ellis D, Winter C, Donald F, et al. (2008) Patientactor perception of care: a comparison of obstetric emergency training using manikins and patient-actors. Quality \& Safety in Health Care 17: 20-24.

17. Chenot JF, Simmenroth-Nayda A, Koch A, Fischer T, Scherer M, et al. (2007) Can student tutors act as examiners in an objective structured clinical examination? Medical Education 41: 1032-1038.

18. Luck J, Peabody JW (2002) Using standardised patients to measure physicians' practice: validation study using audio recordings. BMJ (Clinical Research Ed) 325: 679. 